\title{
Engagement y evolución de instagramers hispanohablantes de moda
}

\author{
Engagement and evolution of Spanish-speaking fashion \\ instagrammers
}

\author{
Erika-Lucia Gonzalez-Carrion. Universidad Nacional de Loja. España. \\ erika.gonzalez@,unl.edu.ec \\ $[\underline{\mathrm{CV}}]$ (D) \\ Ignacio Aguaded. Universidad de Huelva. España. \\ aguaded@uhu.es \\ $[\mathrm{CV}]$ (1) $\mathrm{G}$
}

Este trabajo se ha elaborado en el marco de Alfamed (Red Interuniversitaria Euroamericana de Investigación en Competencias Mediáticas para la Ciudadania), con el apoyo del Proyecto I+D I (2019-2021), titulado "Youtubers e Instagrammers: La competencia mediática en los prosumidores emergentes" con clave RTI2018-093303-B-I00, financiado por Ministerio de Ciencia, Innovación y Universidades de España y el Fondo Europeo de Desarrollo Regional (FEDER).

Cómo citar este artículo / Referencia normalizada

Gonzalez-Carrion, E. L. y Aguaded, I. (2020). Engagement y evolución de instagramers hispanohablantes de moda. Revista Latina de Comunicación Social, (77), 231-252. https://www.doi.org/10.4185/RLCS-2020-1456

\begin{abstract}
RESUMEN
Introducción: Con el auge tecnológico y el despliegue de dispositivos digitales, el fenómeno instagramer se convierte en una revolución global, produciendo un cambio del paradigma comunicativo, del que América Latina y España no quedan exentas; se requiere un análisis respecto al compromiso de los usuarios en la participación e interacción con los contenidos online. Metodología: Principalmente cuantitativa-descriptiva, con extracción de datos por medio de las herramientas de monitoreo SocialBlade y Fan Page Karma, que facilitan información sobre la actividad y engagement de los influencers en Instagram y las principales variables de interacción. Estudio transeccional del mes de febrero (2018, 2019 y 2020) en diez cuentas de instagramers hispanohablantes de moda (América Latina-España). Resultados: El nivel de engagement de las instagramers latinoamericanas es prominente en comparación al español. No existe relación entre el número de seguidores y el compromiso registrado para ambos casos, se observan variaciones destacables y tendencias similares en el sector de la moda. Conclusiones: Las instagramers hispanohablantes presentan una comunidad que suma millones con una tendencia descendente en cuanto a interacción con los contenidos, base del compromiso con sus seguidores, con nuevas correlaciones entre su capacidad para influir y el esfuerzo dedicado en Instagram.
\end{abstract}

PALABRAS CLAVE: redes sociales; instagramer; cultura digital; Instagram; compromiso; moda; influencer. 


\begin{abstract}
Introduction: With the technological boom and the deployment of digital devices, the influencer phenomenon has become a world revolution, and a change in the communicative paradigm from which Latin America and Spain are not exempt, which requires an engagement analysis of the users in the participation and interaction with the online contents. Methodology: Mainly quantitative and descriptive with data extraction through monitoring tools Social Blade and Fan Page Karma, which facilitate the information focused on the activity and engagement of influencers on Instagram, as well as the main variables of interaction. Transectional study from February in 2018, 2019 and 2020 in 10 accounts from fashion Spanish-speaking instagrammers (Latin America-Spain). Results: The engagement level from Latin American instagrammers is prominent towards the Spanish. It is not kept a relation between the number of followers and the engagement registered in both cases, where significant variations are observed, but similar trends in the fashion area. Conclusions: The Spanishspeaking instagrammers present a community that comes to millions with a descendant trend in interaction with contents, base of the engagement with their followers, suggesting new correlations between both their ability to influence and the effort devoted in Instagram.
\end{abstract}

KEYWORDS: social media; instagrammer; digital culture; Instagram; engagement; fashion; influencer.

\title{
CONTENIDOS
}

1. Introducción. 1.1. El impacto de Instagram. 1.2. Instagramers: Los nuevos líderes de opinión. 1.3. El engagement entre instagramers y seguidores. 2. Metodología. 2.1. Muestra. 2.2. Recolección y tratamiento de datos. 3. Discusión de los resultados. 3.1. Engagement de instagramers de moda en América Latina. 3.2. Engagement de instagramers de moda en España. 3.3. Ranking global: comparativa de instagramers de moda en América Latina y España. 4. Conclusiones. 5. Referencias.

\section{Introducción}

Actualmente, las redes sociales propician un espacio idóneo para el cibercontacto entre personas y colectivos con hábitos e intereses similares. En estas plataformas, la navegación se desarrolla de forma sencilla y sin coste, aunque algunos autores aluden a la venta implícita de datos e información personal (DeHart et al., 2020; Wu et al., 2017). Sin embargo, el número de personas registradas en estos medios se multiplica cada día. Los recursos que registran proveen al público de una sensación de cercanía que rompe barreras de idiosincrasia, cultura o idioma, potenciando la inherente socialización humana (Ibáñez-Cubillas et al., 2017). Así, plataformas sociales en línea como Facebook, Instagram, YouTube, Twitter o TikTok incorporan un dinamismo en los procesos de comunicación que sumergen al usuario en nuevas realidades (Ladogina et al., 2020). O, como expresan Capriotti et al. (2019, p. 1095), "es necesario entender a las redes sociales como un instrumento de diálogo e interacción con los públicos". Adicionalmente, las formas de consumo cambiaron de un modelo de práctica y comportamiento analógico a uno digital, transformando las actitudes de los usuarios. Un mundo 2.0 con demandas cada vez más heterogéneas, y ante las cuales los creadores de contenidos particulares e industriales deben responder con agilidad (Hung et al., 2019).

En este contexto, Instagram se posiciona como la plataforma digital con mayor potencial visual y capacidad de transmisión de mensajes a través de la imagen (Thomas et al., 2020). Desde su creación en 2010, acumula millones de adeptos alrededor del mundo, convirtiéndose en uno de los canales de interacción, promoción y difusión más representativos de la esfera digital. Lee et al. (2015) apuntan que, entre las motivaciones que conllevan al uso reiterado de Instagram, se encuentran la interacción social, la captura y el almacenamiento de fotografías, las diferentes formas de expresión 
proporcionadas por la plataforma (hashtags, filtros, IGTV, stories, menciones, etc.) y la participación activa en la vida de otros usuarios. El surgimiento de referentes y/o líderes de opinión (instagramers), no representa un hecho aislado, puesto que su configuración facilita la creación de estrategias que consolidan la concepción de celebridad digital (Niklander et al., 2016). Un rol propio de la era del Big Data, cuyo potencial depende del contenido audiovisual creado y compartido a nivel internacional.

Dado el presente escenario y, atendiendo a las nuevas tendencias de la Sociedad Red, se plantea un estudio preliminar y exploratorio cuyo objetivo es analizar y comparar las variables de interacción y compromiso (engagement) entre instagramers de moda hispanohablantes y seguidores. De este modo, se pretende distinguir un nuevo entorno para la atracción social desde una perspectiva internacional que descubra nuevos formatos de interrelación online, aplicando, de forma complementaria, un estudio prospectivo de su evolución anual. Este aporte, por tanto, mide el grado de compromiso de dos escenarios geográficos diferentes, de manera que facilite, por medio del diseño exploratorio del fenómeno, de una base para la comprensión del impacto y la retroalimentación entre seguidores e instagramers de moda en la actualidad.

\subsection{El impacto de Instagram}

La impresión visual y la expansión de contenidos, base primigenia de Instagram, revelan un destacable interés de la población a nivel global por narrar historias a través de la imagen (Hernández \& Hernández, 2018). Como indican Casaló, Flavián \& Ibáñez-Sánchez (2020), Instagram construye perfiles de liderazgo, por su naturaleza inherentemente visual y la capacidad de transmitir tendencias. El contenido de habla hispana abarca una superficie del 4,9\% mundial. Se produce, así, una estrecha relación entre usuarios y una plataforma principalmente sustentada por dispositivos móviles, ordenadores y tabletas (Openshaw, 2014; IAB, 2019).

El último informe del Global Digital Report de Hootsuite (2020) indica que las cuentas comerciales o de negocios creadas en Instagram ostentan un promedio mensual de crecimiento (seguidores) de $2,5 \%$, un estimado de publicaciones diarias de 1,7 , un porcentaje de fotografías publicadas en los perfiles del $79 \%$, un porcentaje de vídeos del $21 \% \mathrm{y}$, finalmente, un índice de historias de 13,1 al mes.

Instagram, en opinión de Boy \& Uitermark (2017), actúa en función de las imágenes, facilitando a sus usuarios la captura y difusión de contenidos. Adicional y recientemente, la plataforma ha añadido una serie de funciones que, de igual modo, intensifican las interacciones: filtros, stories, GIFs, IGTV, etc. (Woo \& Kim, 2019; Seyfí \& Soydas, 2017), evidenciando una metamorfosis entre tecnologías y nuevos flujos de ciber-comunicación visual como medio de relación humana (Sheldon \& Bryant, 2016).

El formato de plataformas como Instagram permite la creación de medios propios para relacionarse con una audiencia expansiva mediante la construcción de publicaciones, utilizando modalidades visuales y textuales (Rietveld et al., 2020). Instagram, en este sentido, ofrece una variedad de recursos y herramientas audiovisuales, lo que conduce a que las publicaciones sean más vívidas, personales y emocionales (Parmelee \& Roman, 2020). Esta plataforma destaca como una herramienta efectiva de comunicación y marketing para mostrar productos con descripciones visuales (Ponnusamy et al., 2020). Para Ceyhan (2019), se vive una era visual en la que una persona promedio tiene más probabilidades de ver un vídeo u observar una fotografía que leer un texto escrito. El consumo inmediato y a la carta se convierte en una rutina para todos los usuarios, en tanto 
que la demanda de contenidos multiplataforma encuentra un espacio ideal en Instagram, tanto para quien produce como para quien consume.

La compañía de software de redes, Sprout Social (2020), destaca en su último informe que los usuarios de Instagram navegan en la plataforma 53 minutos por día, fuerte indicador de posicionamiento como red de ocio y entretenimiento. Además, se prevé que el índice de usuarios crezca en los próximos años, convirtiéndose también en un complemento idóneo para el marketing electrónico, oferta de nuevos productos y servicios. Por esta razón, surgen referentes digitales que combinan los negocios y el entretenimiento frente a un público dispuesto a consumirlos.

\subsection{Instagramers: los nuevos líderes de opinión}

Bajo estas condiciones se desenvuelven los ya reconocidos instagramers: usuarios con influencia sobre ingentes comunidades, incitando a los diferentes anunciantes a comercializar productos y servicios por medio de su persona, dada la alta capacidad de persuasión y prescripción publicitaria (Sammis et al., 2015). Por su estructura y naturaleza Instagram ya destaca una estética de interés promocional, y en los perfiles de instagramers esta situación se acentúa, dado el negocio y estrategia empresarial que representan para la industria. Como plantean Fernández-Gómez et al. (2018), el influencer se posiciona como un recurso de marketing que entrelaza sus funciones de prescriptor y gestor de marca personal en redes sociales.

Como enuncian Jin \& Ryu (2020), los instagramers exhiben de forma estratégica una amplia gama de fotografías para mostrar sus estilos de vida, promocionar las marcas que les respaldan y compartir sus historias de vida diaria. Las personas o consumidores interesados en un área en particular siguen a estos instagramers como referentes en noticias y tendencias, además de considerarlos personas influyentes (Weismueller et al., 2020). La mayor distinción para un instagramer es el acceso a gran cantidad de información vinculada al índice de usuarios que lo siguen. De modo que a mayor sea su índice de seguidores, mayor será su popularidad (Castillo \& Palma, 2017). Asimismo, para Díaz (2017) esta incidencia sobre la notoriedad, genera que estos influencers compongan mensajes personalizados que, en muchas ocasiones, se derivarán en recomendaciones promocionales.

En cuanto a la audiencia, Instagram presenta una población online diversa, particular, heterogénea y con diferentes grados de interacción. Tiggermann y Zinoviev (2020) consideran que los usuarios, en este sentido, crean sus propios perfiles personales, comparten y distribuyen información, forman relaciones en línea e interactúan con otros en sus redes. Se conduce a un modelo digital donde cada individuo estructura su propio material, participa en conversaciones, retroalimenta diversos contenidos o, simplemente, disfruta de un conglomerado audiovisual afín a él. Dadas las numerosas interacciones suscitadas entre influyente-seguidores y una comunicación interpersonal marcada, estos son percibidos como creíbles, incluso si se relacionan con una marca ligeramente incongruente (Breves et al., 2019). Echegaray (2015) sugiere que la puesta en escena de un espectador que consume y produce a la vez desemboca en una transfiguración del concepto de audiencia. Así, el instagramer abarca un conjunto de características propias del medio en que se viralizan los contenidos (Márquez \& Lanzeni, 2018), despertando dinámicas de interacción con el público de una forma novedosa, colaborativa y narrativa (Sanjuán et al., 2014; Martínez-Sanz \& GonzálezFernández, 2018).

\subsection{El engagement entre instagramers y seguidores}

La sinergia entre Instagram e instagramer presenta un cúmulo de estrategias de diálogo virtual y asincronía con extensas comunidades, que parte de la latente concepción socializadora de la red 
(Candale, 2017). Esta comunicación se realiza de forma inmediata y con un poder de expansión relevante, al punto que la interacción no se fomenta solo entre usuarios pares, sino que permite a personas totalmente diversas emitir sus opiniones o sensaciones, consolidando una red donde la participación no tiene filtro y es ilimitada (Nedra et al., 2019). A partir de estas premisas se define el engagement que, en palabras de Ballesteros-Herencia (2019), se entiende como la manifestación de diferentes representaciones simbólicas, principalmente a través del 'me gusta', 'compartir' y 'comentar'. Y es que, el auge de las redes digitales trajo consigo diversas maneras de crear y forjar vínculos. No basta ya con la creación de contenidos, sino que debe manifestarse una implicación permanente y constante que genere actualizaciones y consolide un verdadero diálogo con los seguidores (Carrasco-Polaino et al., 2018). Como expresaban Tur-Viñes et al. (2018, p. 1214), "el engagement justifica y sustenta un tipo de relación 'especial' que se establece entre algunos consumidores y determinado contenido audiovisual".

En este sentido, Kim et al. (2017) destacan que los perfiles en Instagram que mayor retroalimentación evidencian, son los que también acumulan más seguidores, ya que el incremento de interacción por intereses comunes desemboca en mayor similitud en un grupo de usuarios (homónimos) (Vizcaíno-Verdú et al., 2019). Sustentando este criterio, Castelló-Martínez (2016) argumenta que el surgimiento de nuevos modelos y líderes de opinión multiplica el alcance de sus acciones de comunicación, deviniendo en una reputación digital clave para atraer miles de seguidores y generar una fuerte interacción o engagement. Se define entonces un nuevo concepto en las relaciones humanas y en los aspectos que determinan a una persona como instagramer, centrándose en la popularidad como un punto central y en la personalidad como generadora de la retroalimentación (Csikszentmihalyi, 1998).

De acuerdo con Da-Luz et al. (2017), en este procedimiento el uso de un lenguaje particular entrelazado con los avances tecnológicos configura nuevos significados por medio de elementos semióticos, imágenes, sonidos y otros. Personas influyentes en redes como los instagramers recurren a estos sistemas de expresión para difundir la información y conseguir sus objetivos. El compromiso de los usuarios con un determinado perfil tiene la capacidad para mejorar su reputación y aumentar innovaciones, pues retener, mantener y nutrir a los usuarios es cada vez una tarea más importante y compleja (Zolkepli et al., 2015). Para Cyhan (2019), el hecho de que una audiencia prosumidora sea participativa en un perfil es decisivo para aumentar una percepción positiva. Esto es, mejorar el grado de engagement en una red como Instagram requiere tiempo, posicionamiento, identidad y acciones prediseñadas y ejecutadas en el momento adecuado. Por lo que, este contexto de necesidades interactivas plantea una evaluación del engagement que, en el entorno de la moda y el lifestyle, adquieren mayor significación (McFarlane \& Samsioe, 2020; Schöps et al., 2019; Skjulstad, 2018; Caldeira, 2018).

\section{Metodología}

La presente investigación emplea una metodología cuantitativo-descriptiva por medio de la recolección de datos. Para ello, se combinan dos herramientas de monitoreo, Fan Page Karma, que permite acceder a un análisis detallado sobre los indicadores de rendimiento de un perfil de Instagram (compromiso, interacción, publicaciones y las reacciones efectuadas en las mismas); y SocialBlade, que facilita una analítica a los perfiles seleccionados y establece un posicionamiento global de los mismos. El fin último es analizar las variables de interacción (actividad clave para el engagement entre el instagramer y sus seguidores) y otros recursos comunicativos generados por las cinco instagramers más representativas de América Latina y España, así como para definir las diferencias existentes entre la muestra seleccionada. 
El periodo de análisis abarcó el mes de febrero de 2018, 2019 y 2020. La selección de estas fechas se justifica en la nula implicación de eventos o actividades coyunturales que puedan ocasionar alteraciones en la actividad normal de la instagramer de moda. Como precedentes, este estudio exploratorio mediante monitoreo y comparativa se acogió a estudios internacionales relacionados con el objeto de estudio, engagement y evolución de los instagramers (Segarra-Saavedra \& HidalgoMarí, 2018; Padilla \& Oliver, 2018; Pérez \& Luque, 2018; De-Casas-Moreno et al., 2018).

\subsection{Muestra}

La delimitación de la muestra parte de la categorización realizada por Influencer Marketing Hub (recurso líder para medir la influencia en las diferentes plataformas digitales) y el portal estadístico Statista (proveedor internacional de datos sobre consumo en línea).

Dada la relevancia de la población de habla hispana en Instagram y la abundante bibliografía sobre instagramers de moda femenina, se procedió a seleccionar la muestra latinoamericana y española. A partir del 'Top 25 de influencers latinoamericanos en Instagram 2019' de Influencer Marketing Hub (https://bit.ly/2xToZzZ), se escogieron las cinco cuentas de origen latinoamericano con mayor impacto en el sector de la moda. Conjuntamente, se recurrió al portal de estadísticas en línea Statista para categorizar los cinco perfiles en el ámbito de la moda más populares de España en el año 2019 (https://bit.ly/35S9aWK). En ambos casos se descartaron, como criterio de exclusión, aquellas instagramers que no respondieran a la concepción de influencer. Esto es, no se contemplaron en la muestra final perfiles creados por celebridades de la industria cinematográfica, televisiva, moda, etc., sino aquellas que han construido desde la anonimidad su instafama (Jabłońska \& Zajdel, 2020). En consecuencia y, como criterios de inclusión, se definieron perfiles con las siguientes características: a) Cuentas con más de un millón de seguidores; b) Mujeres; c) Sector moda; d) Edad máxima de 35 años; e) América Latina/España.

Tabla 1. Muestra de la investigación.

\begin{tabular}{|l|l|}
\hline \multicolumn{1}{|c|}{ América Latina } & \multicolumn{1}{c|}{ España } \\
\hline Lelé Pons: 23 años. 40,6 millones de seguidores & Aida Domènech: 30 años. 2,8 millones de seguidores \\
\hline Yuya: 27 años. 15,9 millones de seguidores & Paula Gonu: 27 años. 2 millones de seguidores \\
\hline Camila Coelho: 32 años. 8,8 millones de seguidores & Mónica Morán: 20 años. 1,7 millones de seguidores \\
\hline Bethany Mota: 18 años. 4,5 millones de seguidores & María Pombo: 25 años. 1,4 millones de seguidores \\
\hline Mariale Marrero: 29 años. 6,2 millones de seguidores & Rocío Osorno: 32 años. 1,1 millones de seguidores \\
\hline
\end{tabular}

Fuente: Influencer Marketing Hub y Statista.

\subsection{Recolección y tratamiento de datos}

En la primera fase del monitoreo de datos se recurrió a la herramienta Fan Page Karma para extraer variables relacionas directamente con la concepción de engagement durante el mes de febrero de 2018, 2019 y 2020, a fin de establecer diferencias evolutivas en los índices y establecer los parámetros que lo conforman. Las variables seleccionadas y proporcionadas por la herramienta se recogen en la tabla 2.

Tabla 2. Variables de evaluación del engagement.

\begin{tabular}{|l|l|}
\hline \multicolumn{1}{|c|}{ Variable } & \multicolumn{1}{c|}{ Descripción } \\
\hline Compromiso (engagement) & Porcentaje de compromiso adquirido en el mes de análisis \\
\hline Seguidores & Número de seguidores registrados en el mes de análisis \\
\hline Interacción publicaciones & Porcentaje de participación de los usuarios en los contenidos \\
\hline
\end{tabular}


RLCS, Revista Latina de Comunicación Social, 77, 231-252

[Investigación] DOI: 10.4185/RLCS-2020-1456 | ISSN 1138-5820 | Año 2020

\begin{tabular}{|l|l|}
\hline Evolución semanal & Porcentaje de la evolución semanal en relación con la actividad \\
\hline Índice de rendimiento & Efectividad del perfil y las publicaciones \\
\hline $\mathrm{N}^{\circ}$ de 'me gusta' & Cantidad de 'me gusta' obtenidos en total en el mes \\
\hline $\mathrm{N}^{\mathrm{a}}$ de publicaciones & Cantidad de publicaciones diarias que efectúa cada instagramer \\
\hline Publicaciones por día & Estimación de publicaciones diarias que efectúa cada instagramer \\
\hline $\mathrm{N}^{\circ}$ de comentarios & Total de comentarios realizados en el mes \\
\hline $\mathrm{N}^{\circ}$ total de reacciones & Reacciones del usuario en un mes, en función del contenido que recibe \\
\hline
\end{tabular}

Fuente: Fan Page Karma.

En segunda fase se aplicó la plataforma SocialBlade para establecer una posición de las instagramers en el contexto global, de acuerdo con las variables de la tabla 3.

Tabla 3. Variables de evaluación del posicionamiento global en función del engagement.

\begin{tabular}{|l|l|}
\hline \multicolumn{1}{|c|}{ Variable } & \multicolumn{1}{c|}{ Descripción } \\
\hline $\mathrm{N}^{\mathrm{o}}$ de seguidores & Posicionamiento global de la instagramer en función de los seguidores \\
\hline $\mathrm{N}^{\mathrm{o}}$ de cuentas que sigue & Posicionamiento global de la instagramer en función del $\mathrm{n}^{\circ}$ cuentas que sigue \\
\hline Compromiso (engagement) & Posicionamiento global de la instagramer en función de la interacción \\
\hline Rango en medios & Posicionamiento global de la instagramer en función de la influencia del perfil \\
\hline Calificación del perfil & $\begin{array}{l}\text { Calificación asignada por SocialBlade con base en el éxito, interacción e } \\
\text { influencia de un perfil, donde la puntuación más alta es A++ y la más baja D-. }\end{array}$ \\
\hline
\end{tabular}

Fuente: SocialBlade.

Mediante este sistema se inició el estudio de comparación del impacto en materia de engagement en América Latina y España.

\section{Discusión de los resultados}

\subsection{Engagement de instagramers de moda en América Latina}

El estudio parte de la evaluación de un mes completo (febrero) en 2018, 2019 y 2020. Como se observa en los datos de 2018 (tabla 4), las principales instagramers de América Latina evidencian un engagement elevado, cuyo porcentaje máximo corresponde a Mariale Marrero (25\%), y cuyo porcentaje mínimo a Yuya $(0,75 \%)$. El resto de cuentas mantienen un engagement que oscila entre el $0,93 \%$ y el $9,5 \%$.

Respecto a los seguidores, Lelé Pons se consolida como la instagramer con mayor injerencia en la plataforma, en tanto que convoca 22,7 millones de usuarios, mientras que Mariale Marrero (que presenta el mejor valor de engagement de los perfiles seleccionados), dispone de la menor cantidad de seguidores (3,8 millones). Yuya, Camila Coelho y Bethany Mota abarcan una cuantía que fluctúa entre los 11,2 y 5,5 millones respectivamente.

Tabla 4. Engagement e interacción en febrero de 2018: Instagramers latinoamericanas.

\begin{tabular}{|l|r|r|r|r|r|}
\hline \multicolumn{1}{|c|}{ Instagramer } & Compromiso & Seguidores & $\begin{array}{c}\text { Interacción de } \\
\text { publicaciones }\end{array}$ & $\begin{array}{c}\text { Evolución } \\
\text { semanal }\end{array}$ & $\begin{array}{c}\text { Índice de } \\
\text { rendimiento }\end{array}$ \\
\hline Lelé Pons & $9,5 \%$ & 22,7 millones & $10 \%$ & $0,86 \%$ & $45 \%$ \\
\hline Yuya & $0,75 \%$ & 11,2 millones & $7 \%$ & $0,38 \%$ & $8 \%$ \\
\hline Camila Coelho & $4,2 \%$ & 7 millones & $1,4 \%$ & $0,14 \%$ & $12 \%$ \\
\hline Bethany Mota & $0,93 \%$ & 5,5 millones & $3,7 \%$ & $-0,18 \%$ & $3,0 \%$ \\
\hline Mariale Marrero & $25 \%$ & 3,8 millones & $19 \%$ & $1,3 \%$ & $89 \%$ \\
\hline
\end{tabular}


RLCS, Revista Latina de Comunicación Social, 77, 231-252

[Investigación] DOI: 10.4185/RLCS-2020-1456 | ISSN 1138-5820 | Año 2020

\begin{tabular}{|l|r|r|r|r|r|}
\hline \multicolumn{1}{|c|}{ Instagramer } & \multicolumn{1}{|c|}{$\begin{array}{c}\text { Número } \\
\text { de me gusta }\end{array}$} & $\begin{array}{c}\text { Interacción } \\
\text { publicaciones }\end{array}$ & $\begin{array}{c}\text { Publicaciones } \\
\text { por día }\end{array}$ & $\begin{array}{c}\text { Número de } \\
\text { comentarios }\end{array}$ & $\begin{array}{c}\text { Total de } \\
\text { reacciones }\end{array}$ \\
\hline Lelé Pons & 58,9 millones & 26 & 0,9 & 717 mil & 59,6 millones \\
\hline Yuya & 2,2 millones & 3 & 0,1 & $123 \mathrm{mil}$ & 2,3 millones \\
\hline Camila Coelho & 8,3 millones & 88 & 3,1 & 717 mil & 8,3 millones \\
\hline Bethany Mota & 1,4 millones & 7 & 0,3 & 5,7 mil & 1,4 millones \\
\hline Mariale Marrero & 21,7 millones & 37 & 1,3 & 4,4 mil & 26,1 millones \\
\hline
\end{tabular}

Fuente: Fan Page Karma.

Por otra parte, la interacción que las instagramers producen no sobrepasa el 19\%. Tal es el caso de Mariale Marrero. De hecho, la mínima interacción en un perfil la presenta Camila Coelho con un 1,4\%. Las instagramers restantes oscilan entre un índice de interacción del 3\% al 10\%. La evolución semanal e índice de rendimiento total del perfil posiciona a dos instagramers como las influencers con mayor productividad en sus cuentas: Mariale Marrero (89\%) y Lelé Pons (45\%), convirtiéndose en referentes del sector.

Igualmente, y, en referencia al intercambio y acciones de los usuarios, Lelé Pons y Mariale Marrero reiteran su posicionamiento con la mayor cantidad de 'me gustas' acumulados (58,9 y 21,7 millones respectivamente). Los otros tres perfiles se encuentran por debajo de los diez millones de 'me gustas' en el tiempo analizado (entre 1,4 y 8,3 millones). Se observa una relación directa entre número de publicaciones e intercambio con la audiencia, donde tres instagramers introducen más publicaciones en sus cuentas: Camila Coelho (88 publicaciones, más de dos al día), Mariale Marrero (37 publicaciones, más de una al día) y Lelé Pons (26 publicaciones, mayoritariamente una al día).

Finalmente, el número de comentarios y reacciones muestran el gran distanciamiento que existe entre las instagramers de la misma extensión geográfica. Estos datos ubican como perfil principal a Lelé Pons (con una estimación de 717.000 comentarios y 59.6 millones de reacciones en un mes), Yuya (123.000 comentarios y 2,1 millones de reacciones en un mes) y Mariale Marrero (con 4.400 comentarios y 26,1 millones de reacciones en un mes). Se ha de atender que las instagramers obtienen rangos muy elevados de interacción en un periodo relativamente reducido (un mes), lo que da cuenta de su alcance e influencia dentro de un medio digital.

Tabla 5. Engagement e interacción en febrero de 2019.

\begin{tabular}{|c|c|c|c|c|c|}
\hline Instagramer & Compromiso & Seguidores & $\begin{array}{l}\text { Interacción de } \\
\text { publicaciones }\end{array}$ & $\begin{array}{c}\text { Evolución } \\
\text { semanal } \\
\end{array}$ & $\begin{array}{l}\text { Índice de } \\
\text { rendimiento }\end{array}$ \\
\hline Lelé Pons & $5,1 \%$ & 33,4 millones & $4,7 \%$ & $0,58 \%$ & $27 \%$ \\
\hline Yuya & $2,7 \%$ & 13,6 millones & $4,9 \%$ & $0,28 \%$ & $14 \%$ \\
\hline Camila Coelho & $3,0 \%$ & 8,1 millones & $1,5 \%$ & $0,11 \%$ & $10 \%$ \\
\hline Bethany Mota & $0,50 \%$ & 5,1 millones & $3,5 \%$ & $-0,18 \%$ & $2 \%$ \\
\hline $\begin{array}{l}\text { Mariale } \\
\text { Marrero }\end{array}$ & $5,7 \%$ & 5,5 millones & $4,8 \%$ & $0,47 \%$ & $26 \%$ \\
\hline \multicolumn{6}{|c|}{ Interacción } \\
\hline Instagramer & $\mathrm{N}^{\circ}$ de me gusta & $\begin{array}{c}\mathrm{N}^{\circ} \text { de } \\
\text { publicaciones }\end{array}$ & $\begin{array}{c}\text { Publicaciones } \\
\text { por día }\end{array}$ & $\begin{array}{l}\text { Número de } \\
\text { comentarios }\end{array}$ & $\begin{array}{c}\text { Total de } \\
\text { reacciones }\end{array}$ \\
\hline Lelé Pons & 46,6 millones & 30 & 1,1 & $406 \mathrm{~K}$. & 47 millones \\
\hline Yuya & 10 millones & 15 & 0,5 & $55 \mathrm{~K}$ & 10,1 millones \\
\hline Camila Coelho & 6,2 millones & 58 & 2,1 & $43 \mathrm{~K}$. & 6,3 millones \\
\hline Bethany Mota & $712 \mathrm{mil}$ & 4 & 0,1 & $3 \mathrm{~K}$. & $715 \mathrm{mil}$ \\
\hline $\begin{array}{l}\text { Mariale } \\
\text { Marrero }\end{array}$ & 8 millones & 33 & 1,2 & $796 \mathrm{~K}$. & 8,7 millones \\
\hline
\end{tabular}

Fuente: Fan Page Karma. 
En 2019 se observa una variación en cuanto a los índices que ostenta cada instagramer. No obstante, Mariale Marrero y Lelé Pons continúan marcando un liderazgo entre los perfiles de moda analizados, con un engagement de 5,7\% y 5,1\% respectivamente (rango notablemente menor al 2018) y convirtiéndose en los perfiles más destacados. El resto se encuentra entre el 0,93\% y 4,2\%, marcando una tendencia descendente respecto del año previo. En cuanto al número de seguidores que se registra en cada cuenta, todas las instagramers aumentaron su audiencia en la plataforma. Por ejemplo, Lelé Pons alcanzó los 34,4 millones y Yuya los 13,6 millones, mientras las demás captaron entre 5,1 y 8,1 millones de seguidores.

La interacción de estas cuentas también presenta un debilitamiento respecto al año anterior, resaltando tres instagramers: Yuya, Lelé Pons y Mariale Barrero, donde no se sobrepasa el umbral del 4,9\% (en 2018 la instagramer con mejor interacción llegó al 19\%). En cuanto a la evolución semanal y al índice de rendimiento de la página, también se produjeron bajas significativas. Las más sobresalientes son las de Lelé Pons (27\%) y Mariale Marrero (26\%). De igual forma, el resto de instagramers también evidenciaron valores negativos que rondan el $2 \%$ y el $14 \%$.

La participación con los usuarios presenta algunas variaciones respecto al 2018, particularmente en la disminución de las acciones efectuadas por la audiencia (aunque en las instagramers más destacadas aún sigue siendo representativa). De esta forma, se consolidan como líderes Lelé Pons y Yuya con 46,6 millones de 'me gustas' y 10 millones de 'me gustas' en el periodo de análisis. Las tres cuentas restantes abarcan un índice que oscila desde los 712.000 'me gustas' a los 8 millones, cifras por debajo de los datos recogidos en el 2018. En esta línea, la continuidad de las publicaciones en algunos casos se incrementa y en otros disminuye, ocupando los primero lugares Camila Coelho (58 publicaciones, más de dos por día), Mariale Marrero (33 publicaciones, más de una por día) y Lelé Pons (30 publicaciones, una por día), reflejando que el número de publicaciones no siempre determina el compromiso establecido con el público.

Consecuentemente, el número de comentarios y el total de reacciones generadas en los perfiles presentan disminuciones respecto a los datos previos. Pese a esto, vuelven a ocupar los primeros lugares las instagramers Lelé Pons (406.000 comentarios y un total de 47 millones de reacciones) y Mariale Marrero (796.000 comentarios y 8,7 millones reacciones). Únicamente Yuya se acerca a ellas con una estimación de 55.000 reacciones y 10,1 millones de reacciones. El resto captó valores por debajo de estas referencias.

Tabla 6. Engagement e interacción en febrero de 2020.

\begin{tabular}{|c|c|c|c|c|c|}
\hline Instagramer & Compromiso & Seguidores & $\begin{array}{l}\text { Interacción de } \\
\text { publicaciones }\end{array}$ & $\begin{array}{c}\text { Evolución } \\
\text { semanal }\end{array}$ & $\begin{array}{c}\text { Índice de } \\
\text { rendimiento }\end{array}$ \\
\hline Lelé Pons & $3,0 \%$ & 39,3 millones & $5,8 \%$ & $0,31 \%$ & $15 \%$ \\
\hline Yuya & $2,6 \%$ & 15,8 millones & $4,1 \%$ & $0,46 \%$ & $17 \%$ \\
\hline Camila Coelho & $2,1 \%$ & 8,7 millones & $1,2 \%$ & $0,19 \%$ & $10 \%$ \\
\hline Bethany Mota & $0,18 \%$ & 4,9 millones & $2,6 \%$ & $-0,077 \%$ & $1,0 \%$ \\
\hline $\begin{array}{l}\text { Mariale } \\
\text { Marrero }\end{array}$ & $2,3 \%$ & 6,2 millones & $3,4 \%$ & $0,17 \%$ & $10 \%$ \\
\hline \multicolumn{6}{|c|}{ Interacción } \\
\hline Instagramer & $\mathrm{N}^{\circ}$ de me gusta & $\begin{array}{c}\mathrm{N}^{\circ} \mathrm{de} \\
\text { publicaciones }\end{array}$ & $\begin{array}{c}\text { Publicaciones } \\
\text { por día }\end{array}$ & $\begin{array}{l}\text { Número de } \\
\text { comentarios }\end{array}$ & $\begin{array}{c}\text { Total de } \\
\text { reacciones }\end{array}$ \\
\hline Lelé Pons & 33,6 millones & 15 & 0,5 & $177 \mathrm{mil}$ & 33,7 millones \\
\hline Yuya & 11,6 millones & 18 & 0,6 & $31 \mathrm{mil}$ & 11,6 millones \\
\hline Camila Coelho & 5,3 millones & 52 & 1,8 & $35 \mathrm{mil}$ & 5,3 millones \\
\hline Bethany Mota & $249 \mathrm{mil}$ & 2 & 0,07 & 763 & $250 \mathrm{mil}$ \\
\hline $\begin{array}{l}\text { Mariale } \\
\text { Marrero }\end{array}$ & 3,5 millones & 20 & 0,6 & $723 \mathrm{mil}$ & 4,2 millones \\
\hline
\end{tabular}

Fuente: Fan Page Karma. 
En febrero de 2020 (tabla 6) se muestra una clara tendencia de disminución en el compromiso de las instagramers, minorando aún más sus valores en comparación con 2019. En este caso, sigue a la cabeza Lelé Pons con un compromiso del 3\% y, a partir de este punto, se observan índices relativamente similares entre tres instagramers (Yuya, Mariale Marrero y Camila Coelho) con porcentajes entre 2,1\% y 2,6\%. Bethany Mota continúa con un rango notablemente inferior, alcanzando solo el $0,18 \%$ del engagement. En relación con los seguidores, se detecta que todos los perfiles aumentan el número de la comunidad (liderando Lelé Pons y Yuya), a excepción de Bethany Mota, que disminuye de 5,1 millones a 4,9 millones.

Todas las instagramers, menos Lelé Pons, experimentan menores índices en la interacción de sus publicaciones, situación que ya se manifestó al comparar los años 2018 y 2019. Así, los valores de la interacción se ubican entre 5,8\% el máximo (Lelé Pons) y 1,2\% el mínimo (Camila Coelho). De igual manera, al determinar los porcentajes alcanzados en la evolución semanal y el índice de rendimiento de la página, estos siguen disminuyendo. En este contexto, Yuya y Lelé Pons se ubican en las primeras posiciones con un rendimiento del 17\% y el 15\% respectivamente. Camila Coelho y Mariale Marrero consiguen el mismo rendimiento, 10\%, mientras que Bethany Mota solo llega a un $1 \%$.

La participación de los usuarios a través de 'me gustas' comienza a reducirse, tanto al compararlo con el mes de febrero de 2018, como con el de 2019. Pese a esto y, como se ha venido subrayando, Lelé Pons y Mariale Marrero son las instagramers que tienen el índice de 'me gustas' más significativo (33,6 millones y 11,6 millones). Las demás oscilan entre los 249.000 y los 5,3 millones de 'me gustas'. La regularidad con la que se emite contenido también se ve afectada en relación con los años previos, donde nuevamente Camila Coelho se convierte en el perfil con mayor cantidad de publicaciones (52 publicaciones, casi dos al día), Mariale Marrero (20 publicaciones con contenidos espaciados), Yuya (18 publicaciones espaciadas), Lelé Pons (15 publicaciones irregulares). Bethany Mota refleja la menor cantidad de actividad con apenas dos publicaciones en todo el mes de febrero.

En el número total de reacciones, que abarca más allá de los 'me gustas' e incluye todo tipo de contenido compartido, se visualiza que, dentro de las instagramer de moda latinas, Lelé Pons se diferencia de las demás, con un promedio de 177.000 comentarios y 33,7 millones de reacciones (estadística superior al de sus coetáneas). Los perfiles que se aproximan a este posicionamiento son el de Mariale Marrero (723.000 comentarios y 4,2 millones de reacciones) y Yuya (31.000 comentarios y 11,6 millones de reacciones). Sin embargo, se ha de destacar que Camila Coelho logra un número de reacciones de entre 5,3 millones que la posiciona como referente en la región.

\subsection{Engagement de instagramers de moda en España}

En el caso de las instagramers españolas, se muestran índices notoriamente inferiores a los casos latinoamericanos. En febrero de 2018 (tabla 7), el engagement alcanzado (a excepción de un perfil) no exponen grandes diferencias. Aida Domènech (Dulceida) se posiciona como la instagramer de moda con mayor compromiso (15\%), seguida de María Pombo (12\%) y Rocío Osorno (10\%). Las dos instagramers restantes, Paula Gonu y Mónica Morán, obtienen un 8,2\% y 0,5\%. En cuanto a seguidores, lideran Aida Domènech y Paula Gonu con 2,2 millones y 1,7 millones respectivamente; el número de seguidores que acumulan las demás instagramers en este periodo de tiempo fluctúa entre 603.000 y 622.000 seguidores. 
RLCS, Revista Latina de Comunicación Social, 77, 231-252

[Investigación] DOI: 10.4185/RLCS-2020-1456 | ISSN 1138-5820 | Año 2020

Tabla 7. Engagement e interacción en febrero de 2018.

\begin{tabular}{|l|r|r|r|r|r|}
\hline Instagramer & Compromiso & Seguidores & $\begin{array}{c}\text { Interacción de } \\
\text { publicaciones }\end{array}$ & $\begin{array}{c}\text { Evolución } \\
\text { semanal }\end{array}$ & $\begin{array}{c}\text { Índice de } \\
\text { rendimiento }\end{array}$ \\
\hline $\begin{array}{l}\text { Aida } \\
\text { Domènech }\end{array}$ & $15 \%$ & 2,2 millones & $6,4 \%$ & $0,39 \%$ & $38 \%$ \\
\hline Paula Gonu & $8,2 \%$ & 1,7 millones & $15 \%$ & $0,21 \%$ & $21 \%$ \\
\hline Mónica Morán & $0,5 \%$ & $622 \mathrm{mil}$ & $1,1 \%$ & $0,14 \%$ & $18 \%$ \\
\hline María Pombo & $12 \%$ & $723 \mathrm{mil}$ & $8,9 \%$ & $0,89 \%$ & $51 \%$ \\
\hline Rocío Osorno & $10 \%$ & \multicolumn{6}{|c|}{603 mil } \\
\hline \multicolumn{7}{|c|}{ Interacción } \\
\hline Instagramer & $\mathbf{N}^{\circ}$ de me gusta & $\begin{array}{c}\text { No de } \\
\text { publicaciones }\end{array}$ & $\begin{array}{c}\text { Publicaciones } \\
\text { por día }\end{array}$ & $\begin{array}{c}\text { Número de } \\
\text { comentarios }\end{array}$ & $\begin{array}{c}\text { Total de } \\
\text { reacciones }\end{array}$ \\
\hline $\begin{array}{l}\text { Aida } \\
\text { Domènech }\end{array}$ & 9,2 millones & 64 & 2,3 & 42 mil & 9,2 millones \\
\hline Paula Gonu & 3,7 millones & 15 & 0,5 & 58 mil & 3,8 millones \\
\hline Mónica Morán & 1,3 millones & 11 & 0,4 & 27 mil & 1,3 millones \\
\hline María Pombo & 2,3 millones & 37 & 1,3 & 57 mil & 2,3 millones \\
\hline Rocío Osorno & 1,4 millones & 42 & 1,5 & 207 mil & 1,6 millones \\
\hline
\end{tabular}

Fuente: Fan Page Karma.

La interacción de las cuentas refleja que Paula Gonu y María Pombo obtienen los porcentajes más elevados, con $15 \%$ y $8,9 \%$ de interacción. A partir de aquí las demás instagramers fluctúan entre un mínimo del $1,1 \%$ y un máximo del $6,9 \%$. La evolución semanal y el índice de rendimiento de los diferentes perfiles señala que las instagramers con mantenimiento del perfil son Rocío Osorno (79\%), pese a no acumular la mayor cantidad de seguidores; y María Pombo (51\%). Las otras cuentas, si bien no son mínimos los porcentajes, descienden a índices de entre los $18 \%$ y $38 \%$ de rendimiento.

Ahora y, particularmente en los 'me gustas' que acumula cada instagramer, es notable la influencia de Aida Domènech sobre los otros perfiles, con 9,2 millones de 'me gustas'. Las cuatro instagramers que le siguen oscilan entre 1,3 millones y 3,7 millones de 'me gustas'. Se observa que fue Aida Domènech la instagramer que mayor cantidad de publicaciones realizó en el tiempo de estudio (64 publicaciones, más de dos por día), seguida de Rocío Osorno (42 publicaciones, más de una publicación por día) y María Pombo (37 publicaciones, más de una por día). Las demás instagramers presentan un número de publicaciones por debajo de las 15 , lo que supone una continuidad escasa en los contenidos que presentan a su comunidad.

Por lo que respecta a los 'me gustas', se evidencia una tendencia similar en el total de comentarios y reacciones acumuladas por instagramer. Aída Domenech lidera el listado con 9,2 millones de reacciones y una estimación de 42.000 comentarios, marcando una distancia notable con el resto de los perfiles. Le siguen Paula Gonu (58.000 comentarios y 3,8 millones de reacciones), María Pombo (57.000 comentarios y 2,3 millones de reacciones), Rocío Osorno (207.000 comentarios y 1,6 millones de reacciones) y Mónica Morán (27.000 comentarios y 1,3 millones de reacciones).

Tabla 8. Engagement e interacción en febrero de 2019.

\begin{tabular}{|l|r|r|r|r|r|}
\hline Instagramer & Compromiso & Seguidores & $\begin{array}{c}\text { Interacción de } \\
\text { publicaciones }\end{array}$ & $\begin{array}{c}\text { Evolución } \\
\text { semanal }\end{array}$ & $\begin{array}{c}\text { Índice de } \\
\text { rendimiento }\end{array}$ \\
\hline $\begin{array}{l}\text { Aida } \\
\text { Domènech }\end{array}$ & $7,4 \%$ & 2,5 millones & $5,3 \%$ & $0,32 \%$ & $33 \%$ \\
\hline Paula Gonu & $13 \%$ & 1,9 millones & $13 \%$ & $0,17 \%$ & $19 \%$ \\
\hline
\end{tabular}


RLCS, Revista Latina de Comunicación Social, 77, 231-252

[Investigación] DOI: 10.4185/RLCS-2020-1456 | ISSN 1138-5820 | Año 2020

\begin{tabular}{|l|r|r|r|r|r|}
\hline Mónica Morán & $2,5 \%$ & 1,2 millones & $16 \%$ & $0,42 \%$ & $15 \%$ \\
\hline María Pombo & $12 \%$ & $996 \mathrm{~K}$. & $7,8 \%$ & $0,43 \%$ & $36 \%$ \\
\hline Rocío Osorno & $11 \%$ & \multicolumn{5}{|c|}{$950 \mathrm{~K}}$. & \multicolumn{2}{c|}{$8,7 \%$} & $0,70 \%$ & $43 \%$ \\
\hline \multicolumn{7}{|c|}{ Interacción } \\
\hline Instagramer & $\mathbf{N}^{\circ}$ de me gusta & $\begin{array}{c}\mathbf{N}^{\circ} \text { de } \\
\text { publicaciones }\end{array}$ & $\begin{array}{c}\text { Publicaciones } \\
\text { por día }\end{array}$ & $\begin{array}{c}\text { Número de } \\
\text { comentarios }\end{array}$ & $\begin{array}{c}\text { Total de } \\
\text { reacciones }\end{array}$ \\
\hline $\begin{array}{l}\text { Aida } \\
\text { Domènech }\end{array}$ & 4,6 millones & 39 & 1,4 & 20 mil & 4,7 millones \\
\hline Paula Gonu & 5,1 millones & 28 & 1,0 & 70 mil & 5,2 millones \\
\hline Mónica Morán & 2 millones & 10 & 0,4 & 22 mil & 2 millones \\
\hline María Pombo & 3,3 millones & 43 & 1,5 & 17 mil & 3,3 millones \\
\hline Rocío Osorno & 2,2 millones & 34 & 1 & 539 mil & 2,8 millones \\
\hline
\end{tabular}

Fuente: Fan Page Karma.

En 2019 se producen algunos cambios en las instagramers de moda españolas (tabla 8). Tres de ellas incrementan sus niveles de engagement: Paula Gonu alcanza el 13\%, Rocío Osorno el 11\% y Mónica Morán el 2,5\%; mientras que Aida Domènech baja al 7,4\% y María Pombo se mantiene. Como viene siendo una tendencia entre las instagramers de moda, de un año a otro se incrementa el número de seguidores, posicionándose en los primeros lugares Aida Domènech (2,2 millones de seguidores), Paula Gonu (1,9 millones) y Mónica Morán, que presenta uno de los aumentos más significativos, llegando a los 1,2 millones de seguidores. María Pombo y Rocío Osorno abarcan 950.000 o 996.000 cada una.

Respecto a la interacción, durante el mes de análisis se comprueba una reducción en tres de ellas: Aida Domènech con 5,3\%, Paula Gonu con 13\% y María Pombo con 7,8\%. Sin embargo, la instagramer Mónica Morán eleva su nivel de interacción hasta el 16\% y Rocío Osorno incrementa mínimamente sus índices hasta el 8,7\%. En la evolución semanal y el compendio final del índice de rendimiento de los perfiles, la mayoría desciende. Debe resaltarse el rendimiento obtenido por María Pombo (36\%) y Roció Osorno (43\%), que supera a sus índices obtenidos en el 2018 sobre la efectividad total.

Los 'me gustas' que obtienen las instagramers de moda aumentan en 2019 en casi todos los casos, fluctuando entre los 2,2 millones como mínimo y los 5,1 millones como máximo. Solo la instagramer Aida Domènech (pese a ser la que mayor cantidad de seguidores tiene), experimenta un descenso en los 'me gustas' de sus publicaciones, al obtener 4,6 millones, prácticamente la mitad en comparación con 2018. En este grupo, los tres perfiles con mayor cantidad de publicaciones corresponden a María Pombo (43 publicaciones, más de una publicación al día), Aida Domènech (39 publicaciones, más de una publicación al día) y Rocío Osorno (34 publicaciones, generalmente una al día). Paula Gonu y Mónica Morán son las instagramers que menos publican con 28 y 10 publicaciones en todo el mes.

En los comentarios y, particularmente, en las reacciones, se muestra que todas las instagramers aumentan sus índices excepto Aida Domench que, de 9,2 reacciones en 2018, pasa a 4,7 millones en 2019. En el resto de los perfiles destacan Paula Gonu (70.000 comentarios y 5,2 millones de reacciones), María Pombo (17.000 comentarios y 3,3 millones de reacciones) y, finalmente, Mónica Morán y Rocío Osorno con 2 y 2,8 millones de reacciones cada una. 
RLCS, Revista Latina de Comunicación Social, 77, 231-252

[Investigación] DOI: 10.4185/RLCS-2020-1456 | ISSN 1138-5820 | Año 2020

Tabla 9. Engagement e interacción en febrero de 2020.

\begin{tabular}{|c|c|c|c|c|c|}
\hline Instagramer & Compromiso & Seguidores & $\begin{array}{l}\text { Interacción de } \\
\text { publicaciones }\end{array}$ & $\begin{array}{c}\text { Evolución } \\
\text { semanal }\end{array}$ & $\begin{array}{l}\text { Índice de } \\
\text { rendimiento }\end{array}$ \\
\hline $\begin{array}{l}\text { Aida } \\
\text { Domènech }\end{array}$ & $5,1 \%$ & 2,8 millones & $3,7 \%$ & $0,28 \%$ & $30 \%$ \\
\hline Paula Gonu & $2,6 \%$ & 2 millones & $8,4 \%$ & $0,15 \%$ & $17 \%$ \\
\hline Mónica Morán & $2,9 \%$ & 1,5 millones & $17 \%$ & $0,41 \%$ & $12 \%$ \\
\hline María Pombo & $7,2 \%$ & 1,4 millones & $5,9 \%$ & $0,11 \%$ & $14 \%$ \\
\hline Rocío Osorno & $5,0 \%$ & 1,2 millones & $6 \%$ & $0,18 \%$ & $15 \%$ \\
\hline \multicolumn{6}{|c|}{ Interacción } \\
\hline Instagramer & $\mathrm{N}^{\circ}$ de me gusta & $\begin{array}{c}\mathbf{N}^{\circ} \mathrm{de} \\
\text { publicaciones }\end{array}$ & $\begin{array}{l}\text { Publicaciones } \\
\text { por día }\end{array}$ & $\begin{array}{l}\text { Número de } \\
\text { comentarios }\end{array}$ & $\begin{array}{l}\text { Total de } \\
\text { reacciones }\end{array}$ \\
\hline $\begin{array}{l}\text { Aida } \\
\text { Domènech }\end{array}$ & 4 millones & 40 & 1,4 & $13 \mathrm{mil}$ & 4 millones \\
\hline Paula Gonu & 1,4 millones & 9 & 0,3 & $8.8 \mathrm{mil}$ & 1,4 millones \\
\hline Mónica Morán & 1,3 millones & 5 & 0,2 & $16 \mathrm{mil}$ & 1,3 millones \\
\hline María Pombo & 2,9 millones & 35 & 1,2 & 8,3 mil & 2,9 millones \\
\hline Rocío Osorno & 1,2 millones & 24 & 0,8 & $444 \mathrm{mil}$ & 1,7 millones \\
\hline
\end{tabular}

Fuente: Fan Page Karma.

En febrero de 2020 (tabla 9), el engagement de casi todas las instagramers evidencia una disminución notable, con porcentajes que van desde 2,6\% al 7,2\%, siendo Mónica Morán la única que eleva ligeramente sus niveles de compromiso (2,9\%). En cuanto al número de seguidores, todas incrementan sus índices y sobrepasan el millón de fans en su perfil: Aida Domènech y Paula Gonu lideran con 2,8 millones y 2 millones respectivamente, mientras que las demás se encuentran en una media de entre 1,2 y 1,5 millones.

De forma similar, gran parte de las instagramers disminuye su interacción en las publicaciones, plasmando un margen de entre el 5,3\% mínimo (de Aida Domènech), al 16\% máximo (Mónica Morán). Las demás cuentas fluctúan entre el 7,8\% y el 13\% de interacción (datos por debajo de lo expuesto en el año anterior). Las deficiencias en la interacción provocan también que los índices de evolución semanal y el rendimiento se reduzcan, consolidando en el rango más elevado a Aida Domènech (30\%), y en el más disminuido a Mónica Morán (12\%). Las tres instagramers restantes muestran valores cercanos de entre $14 \%$ y $17 \%$.

Por lo que respecta a 'me gustas' en 2020, en todos los casos desciende el número. No obstante, las instagramers que logran resaltar son Aida Domènech con 4 millones de 'me gustas' y María Pombo con 2,9 millones. Para el resto, la cantidad oscila entre 1,2 y 1,4 millones. Se observa que estos resultados mantienen conexión con el número de publicaciones realizadas en el lapso de tiempo estudiado, ubicando en los primeros lugares a las instagramers Aida Domènech (40 publicaciones, más de una por día), María Pombo (35 publicaciones, en algunos casos superando una por día) y Rocío Osorno (24 publicaciones, una por día con irregularidad); Paula Gonu y Mónica Morán realizan únicamente nueve y cinco publicaciones cada una durante el mes.

En este punto y, aunque en algunos se casos se elevan los números de comentarios, el total de reacciones disminuye, indicando un menor índice de contenidos compartidos o transferidos a las historias de Instagram. Aida Domènech (13.000 comentarios y 4 millones de reacciones) y María Pombo (83.000 comentarios y 2,9 millones de reacciones) lideran esta clasificación, mientras que las demás instagramers alcanzan valores similares de entre 1,3 y 1,7 millones de reacciones en total. 
RLCS, Revista Latina de Comunicación Social, 77, 231-252

[Investigación] DOI: 10.4185/RLCS-2020-1456 | ISSN 1138-5820 | Año 2020

\subsection{Ranking global: comparativa de instagramers de moda en América Latina y España}

Partiendo del análisis geográfico, a continuación, se ilustra una comparativa de las variables que describen el engagement de las cuentas de Instagram femeninas de moda en América Latina y España. En la figura $1 \mathrm{y}$, en relación con los criterios porcentuales del compromiso (interacción con las publicaciones, evolución semanal e índice rendimiento), se observa una preponderancia del eje español. Si bien, la variación es mínima, presentando en ambos casos una tendencia similar.

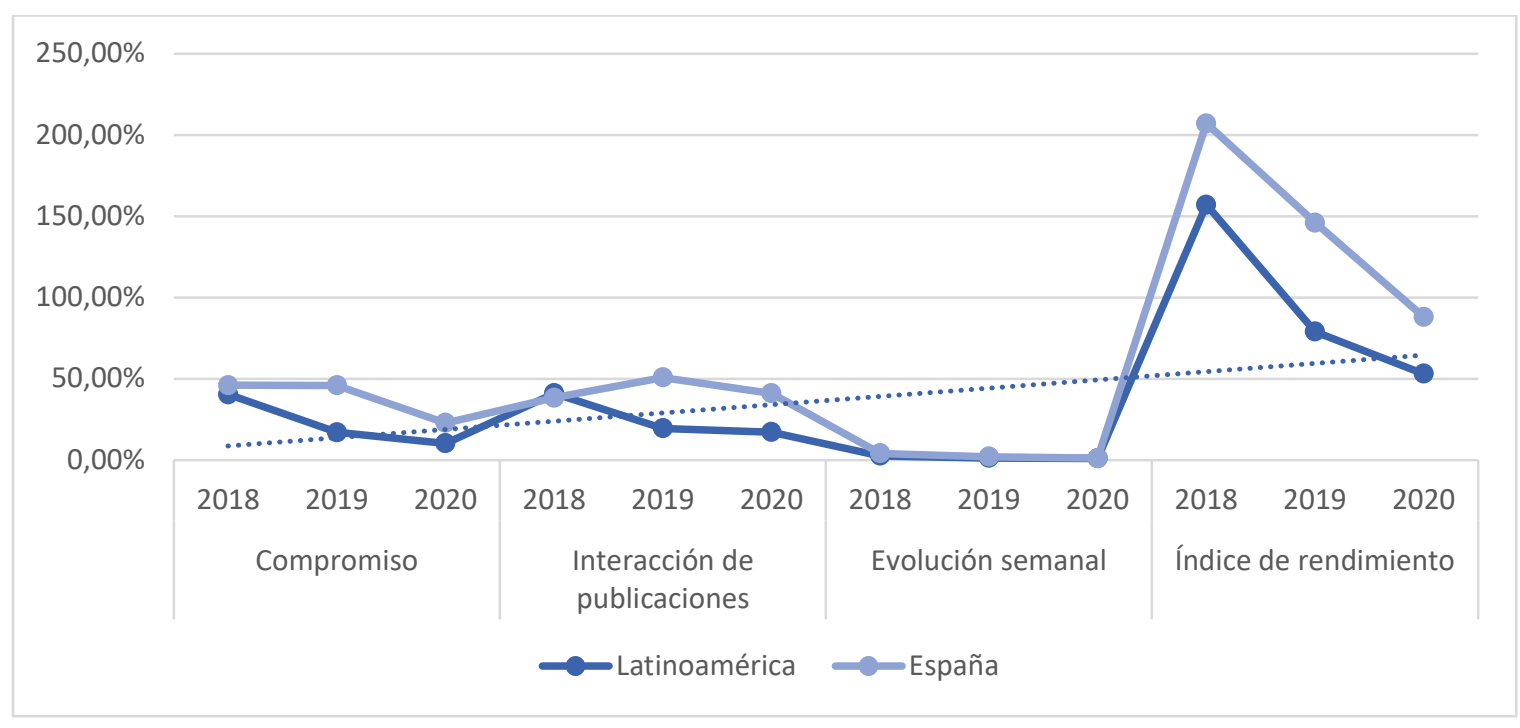

Figura 1: comparativa de variables porcentuales en América Latina y España.

Fuente: elaboración propia.

Para cada caso se observan de forma evolutiva diferentes escenarios. Por ejemplo, tanto en América Latina como en España, el compromiso (engagement) proporcionado por la plataforma es descendente, al igual que el rendimiento o las interacciones en las publicaciones, describiendo una trayectoria constante, pero levemente negativa. En el caso de las variables que se corresponden con aspectos numéricos como los seguidores, 'me gustas', publicaciones, publicaciones por día, comentarios y reacciones, prepondera América Latina con una tendencia irregular (figura 2). Estas fluctuaciones anuales describen un recorrido que, si bien puede estar influenciado por acontecimientos políticos y sociales, difieren en ambos territorios. Por ejemplo, el número de comentarios decrece en el año 2019 en América Latina, mientras que aumenta en España. De hecho, resulta de especial interés indicar que, en el caso de la muestra española, el número de seguidores decae sustancialmente en el año 2020, en concordancia a otros factores como el número de 'me gustas', publicaciones y reacciones. 
RLCS, Revista Latina de Comunicación Social, 77, 231-252

[Investigación] DOI: 10.4185/RLCS-2020-1456 | ISSN 1138-5820 | Año 2020

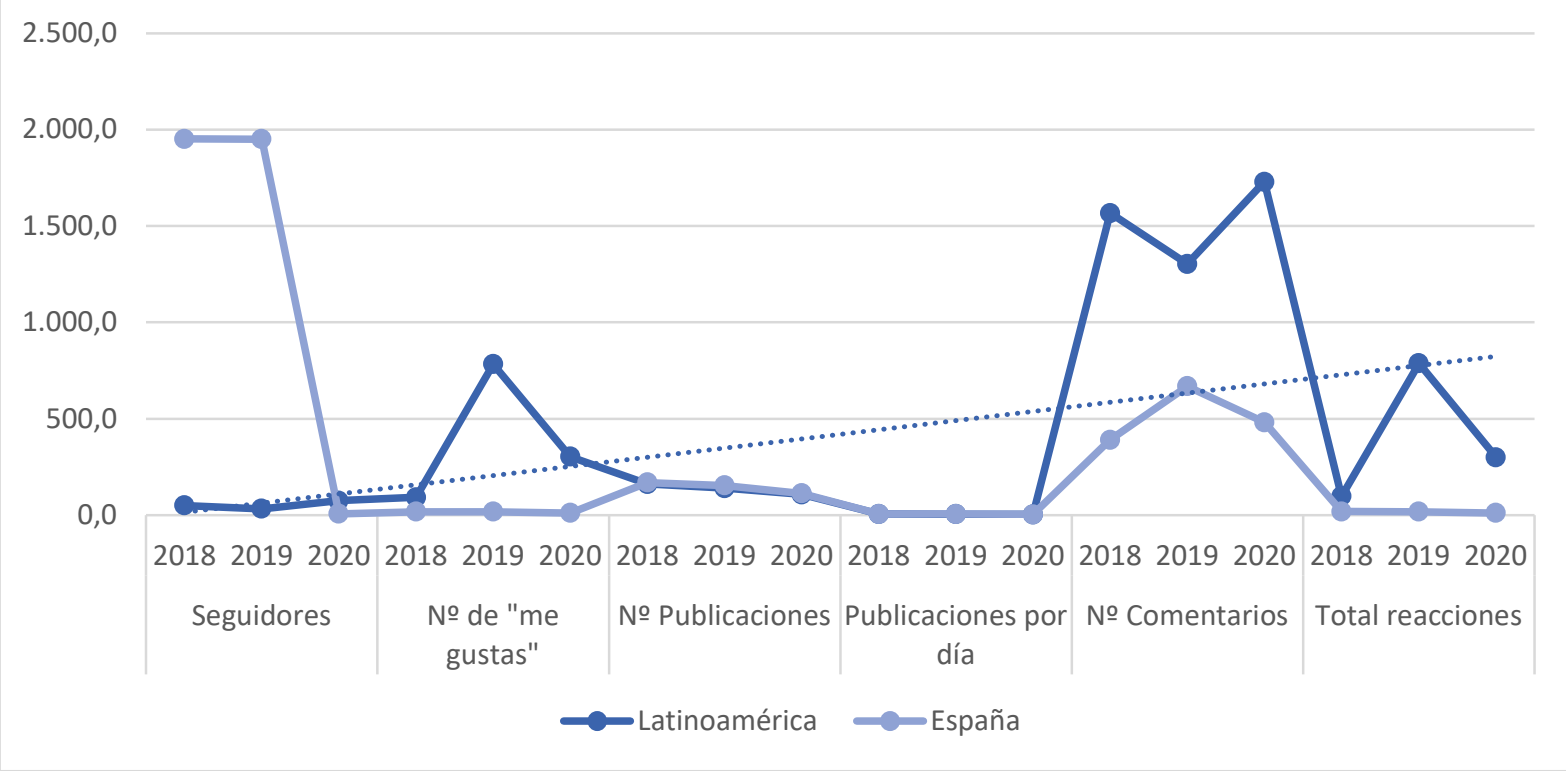

Figura 2: comparativa de variables numéricas en América Latina y España.

Fuente: elaboración propia.

En el marco global y, atendiendo a las clasificaciones proporcionadas por SocialBlade, se observa que la instagramer latinoamericana Lelé Pons se posiciona en primer lugar, ocupando el $\mathrm{n}^{\mathrm{o}} 63$ en la escala mundial de seguidores (tabla 10). La posición más desfavorecedora corresponde a Bethany Mota en el $\mathrm{n}^{\mathrm{o}} 2.075$. Por otra parte, el posicionamiento respecto a cuentas seguidas no es equiparable, en tanto que perfiles sobresalientes como el de la muestra, caracterizados por un elevado nivel de interacciones y engagement, no siguen a un extenso número de usuarios.

Tabla 10. Ranking global: Instagramers latinoamericanas.

\begin{tabular}{|l|r|r|r|r|r|}
\hline Instagramer & $\begin{array}{c}\text { Ranking } \\
\text { global / N } \\
\text { seguido } \mathbf{d e}\end{array}$ & $\begin{array}{c}\text { Ranking global / } \\
\mathbf{N}^{\circ} \text { de cuentas } \\
\text { que sigue }\end{array}$ & $\begin{array}{c}\text { Ranking } \\
\text { global / } \\
\text { Engagement }\end{array}$ & $\begin{array}{c}\text { Rango en } \\
\text { Medios }\end{array}$ & $\begin{array}{c}\text { Calificación } \\
\text { del Perfil }\end{array}$ \\
\hline Lelé Pons & 63 & 1,6 millón & 3,6 millón & 505 mil & $\mathrm{A}$ \\
\hline Yuya & 339 & 4,9 millón & 4,1 millón & 2,5 millón & $\mathrm{A}$ \\
\hline Camila Coelho & 848 & 4,3 millón & 7,2 millón & 31,7 mil & $\mathrm{A}$ \\
\hline Bethany Mota & 2.075 & 5,6 millón & 5,6 millón & 629 mil & A- \\
\hline $\begin{array}{l}\text { Mariale } \\
\text { Marrero }\end{array}$ & 1.432 & 4,6 millón & 5,2 millón & 111 mil & \\
\hline
\end{tabular}

Fuente: SocialBlade.

Respecto al nivel de engagement a nivel mundial, resalta nuevamente Lelé Pons con un valor de 3,6, Yuya con 4,1 y Mariale Marrero con 5,2. En detrimento, las instagramers con menor posición son Bethany Mota y Camila Coelho. Aunque su rango en medios digitales, al igual que el compromiso, las ubica en una posición inferior en el ranking mundial, es destacable señalar que cuasi todas las instagramers de moda latinoamericanas adquieren una calificación A, exceptuando Mariale Marrero (A-). Ello las constituye como referentes digitales. 
RLCS, Revista Latina de Comunicación Social, 77, 231-252

[Investigación] DOI: 10.4185/RLCS-2020-1456 | ISSN 1138-5820 | Año 2020

Tabla 11. Ranking global: Instagramers españolas.

\begin{tabular}{|l|r|r|r|r|r|}
\hline Instagramer & $\begin{array}{c}\text { Ranking } \\
\text { global / N } \\
\text { seguide de }\end{array}$ & $\begin{array}{c}\text { Ranking } \\
\text { global / No de } \\
\text { cuentas que } \\
\text { sigue }\end{array}$ & $\begin{array}{c}\text { Ranking } \\
\text { global } / \\
\text { Engagement }\end{array}$ & $\begin{array}{c}\text { Rango en } \\
\text { Medios }\end{array}$ & $\begin{array}{c}\text { Calificación } \\
\text { del Perfil }\end{array}$ \\
\hline $\begin{array}{l}\text { Aida } \\
\text { Domènech }\end{array}$ & 4.475 & 4,3 millón & 3,5 millón & 55 mil & A- \\
\hline Paula Gonu & 7.475 & 8 millón & 2,5 millón & 507 mil & B+ \\
\hline Mónica Morán & 9.332 & 8,5 millón & 555 mil & 4,1 millón & $\mathrm{B}+$ \\
\hline María Pombo & 11.950 & 5,8 millón & 2,4 millón & 405 mil & $\mathrm{B}+$ \\
\hline Rocío Osorno & 15.825 & 7,6 millón & 1,8 millón & 507 mil & $\mathrm{B}+$ \\
\hline
\end{tabular}

Fuente: SocialBlade.

En el caso de España, se presentan valores que difieren en cuanto al posicionamiento global (tabla 11). La mejor posición la obtiene la instagramer Aida Domènech (Dulceida) en el $n^{0} 4.475$, seguida de Paula Gonu ( ${ }^{\circ}$ 7.475). El resto se sitúa más allá de la posición 9.000. Como se observa y, en referencia a las cuentas que siguen las instagramers españolas, la posición es reducida porque, al igual que en América Latina, existe una diferencia marcada entre número de seguidores y número de cuentas seguidas, que en la mayoría de los casos es bajo.

En materia de compromiso Mónica Morán lidera (555 millones). Le siguen tres instagramers que mantienen puestos cercanos, Rocío Osorno $(1,8)$, María Pombo $(2,4)$ y Paula Gonu $(2,5)$. Aida Domènech, si bien es la instagramer con mayor cantidad de seguidores, presenta un índice de compromiso débil en comparación a sus coetáneas. La calificación de esta muestra presenta un grado inferior respecto a las instagramers latinoamericanas, obteniendo en su mayoría la calificación de $\mathrm{B}+$, que indica la necesidad de mejorar diversos parámetros (interacción y estrategias de comunicación) para mejorar el engagement. En este orden, Aida Domènech es la única instagramer española que consigue A-.

En suma, la presencia mundial de las instragramers hispanohablantes se proyecta esencialmente en la figura latinoamericana, donde las variables relacionadas con el engagement y la interacción revelan un fenómeno comunicacional emergente y de alto reconocimiento e influencia entre los amantes de la moda.

\section{Conclusiones}

Las instagramers en América Latina y España presentan niveles de engagement dispares con tendencias anuales similares, destacando para cuasi la mayoría de los casos el perfil latino en el sector de la moda. Sin embargo, desde el 2018 al 2020 observamos un notorio descenso de los índices evaluados, resultado de una reducción en la interacción suscitada con el público. Con todo, las diez instagramers hispanohablantes analizadas incrementan su comunidad, potenciando año tras año el número de seguidores (inactivos). Esto es, la audiencia digital de estas instagramers no es propensa a dar 'me gustas', compartir o comentar con asiduidad.

Asimismo, existe una conexión directa entre el número publicaciones, el contenido generado por las instagramers y la cantidad de 'me gustas' que obtienen. La frecuencia con la que se exponen las publicaciones representa un punto clave para despertar el interés de los usuarios e incitarlos a ejecutar diferentes movimientos en estos espacios sociales. Si bien, se ha observado una reducción de estas variables en todos los casos a lo largo de tres años. Así, comprobamos la inexistencia de una 
tendencia generalizada que demuestre, mediante un caso de estudio, que las instagramers de moda con mayor cantidad de seguidores son las que mejor engagement registran. Aunque en algunas cuentas estos parámetros guardan relación, en otras el índice de compromiso no está determinado por el posicionamiento en un ranking global.

El índice de comentarios en las publicaciones de las instagramers es representativo. Se observan disminuciones en la comparativa por años, donde la participación de los usuarios presenta cambios, generalmente provocados por el contenido que se publica y las temáticas que se abordan. En tal caso, queda demostrado que toda instagramer de moda mantiene una estrategia para mantener una audiencia y captar nuevos seguidores, misma que se sustenta en un número de publicaciones semanales determinado o un material que genere reacciones masivas.

Con los datos obtenidos, es notable una mejor gestión digital en las instagramers de moda latinas por el movimiento generado en la plataforma Instagram. Destacan su interacción, sus índices de evolución y, principalmente, las reacciones que consiguen con sus publicaciones, conjuntamente con una calificación óptima en un entorno con fuertes grados de competitividad. Así, el rubro de la moda es uno de los campos con mayor influencia entre la comunidad de Instagram, y aún con niveles variados, acumulan un feedback notable para la plataforma.

Por otro lado, y, aunque el nivel de interacciones y clasificaciones globales son notables para el caso latinoamericano y español, la inclinación descendente de las diferentes variables requiere de un estudio pormenorizado que describa, en detrimento de los estudios que resaltaban la relevancia del sector de la moda femenina en Instagram (McFarlane \& Samsioe, 2020; Schöps et al., 2019; Skjulstad, 2018; Caldeira, 2018), la razón por la que el nivel de interacciones se reduce en estos entornos. Entre ellos, el grado de engagement y el rendimiento global de la cuenta. Partiendo del hecho de que estos perfiles adquieren influencia por medio de sus seguidores y el valor de automarca en la plataforma como indicaban Castillo y Palma (2017), se plantea una hipótesis para futuras líneas de investigación. Estos resultados requieren evaluar si la disminución anual del engagement está proporcionalmente ligada al aumento de influencia de la instagramer. Es decir, si los esfuerzos estratégicos de auto-promoción se dirigen exclusivamente a la comunidad de Instagram o, con el tiempo, a otros medios y plataformas debido al grado de celebridad adquirido. Un hecho que contraría la premisa de Díaz (2017) por la que, a mayor notoriedad, mayor configuración de los contenidos para incrementar la interacción (y atracción) con la comunidad de seguidores actuales y potenciales.

Por tanto, el presente estudio, pese a la limitación de la muestra, introduce un preámbulo para el análisis de correlaciones entre el engagement de instagramers de moda hispanohablantes en materia de seguidores-notoriedad y notoriedad-interacción. Al mismo tiempo, plantea un modelo de evaluación de un fenómeno emergente que reconfigura las relaciones de influencia social a razón de las instagramers de moda, ampliable a otros escenarios que ratifiquen la información resultante y que profundicen en las tendencias entre zonas geográficas y sectores temáticos.

\section{Referencias}

Ballesteros-Herencia, C. A. (2019). La representación digital del engagement: Hacia una percepción del compromiso a través de acciones simbólicas. Revista de Comunicación, 18(1), 215-233. https://doi.org/10.26441/RC18.1-2019-A11

Breves, P., Liebers, N., Abt, M. \& Kunze, A. (2019). The perceived fit between Instagram influencers and the endorsed brand: How influencer-brand fit affects source credibility and 
RLCS, Revista Latina de Comunicación Social, 77, 231-252

[Investigación] DOI: 10.4185/RLCS-2020-1456 | ISSN 1138-5820 | Año 2020

persuasive effectiveness. Journal of Advertising Research, 59(4), 440-454. https://doi.org/10.2501/JAR-2019-030

Caldeira, S. P. (2018). "Shop it. Wear it. Gram it.”: A qualitative textual analysis of women's glossy fashion magazines and their intertextual relationship with Instagram. Feminist Media Studies, 20(1), 86-103. https://doi.org/10.1080/14680777.2018.1548498

Candale, C. (2017). Las características de las redes sociales y las posibilidades de expresión abiertas por ellas. La comunicación de los jóvenes españoles en Facebook, Twitter e Instagram. Colindancias, 8, 201-218. https://bit.ly/2YW3Sbx

Capriotti, P., Zeler, I. \& Oliveira, A. (2019). Comunicación dialógica 2.0 en Facebook. Análisis de la interacción en las organizaciones de América Latina. Revista Latina de Comunicación Social, (74), 1094-1113. https://doi.org/10.4185/RLCS-2019-1373

Carrasco-Polaino, R., Villar-Cirujano, E. \& Martín-Cárdaba, M. A. (2018). Activismo y ONG: Relación entre imagen y «engagement» en Instagram. Comunicar, (57), 29-38. https://doi.org/10.3916/C57-2018-03

Casaló, L., Flavián, C. \& Ibáñez-Sánchez, S. (2020). Influencers on Instagram: Antecedents and consequences of opinion leadership. Journal of Business Research, Article in Press, 1-10. https://doi.org/10.1016/j.jbusres.2018.07.005

Castelló-Martínez, A. (2016). El marketing de influencia: Un caso práctico. En I. Zacipa, V. TurViñes y J. Segarra-Saavedra (Eds.), Tendencias publicitarias en Iberoamérica: Diálogo de saberes y experiencias (pp. 49-65). Colección Mundo Digital.

Castillo, J. \& Palma, M. (2017). Identifying influencers of social work within online social networks. Revista Internacional de Trabajo Social y Bienestar, (6), 81-89. https://bit.ly/2WliRsq

Ceyhan, A. (2019). The impact of perception related social media marketing applications on consumers' brand loyalty and purchase intention. Emerging Markets Journal, 9(1), 87-100. https://doi.org/10.5195/emaj.2019.173

Csikszentmihalyi, M. (1998). Finding flow. The psychology of engagement with everyday life. Basic Books.

Da-Luz, A., Caiado, R. \& Da-Fonte, R. (2017). The instagramer and its multissemiotic discourse on the digital social network Instagram. Dialogo das Letras, 6(2), 139-158.

De-Casas-Moreno, P., Tejedor-Calvo, S. \& Romero-Rodríguez, L. M. (2018). Micronarrativas en Instagram: Análisis del storytelling autobiográfico y de la proyección de identidades de los universitarios del ámbito de la comunicación. Prisma Social, (20), 40-57. https://bit.ly/3cr3TYK

DeHart, J., Stell, M. \& Grant, C. (2020). Social media and the scourge of visual privacy. Information, 11(2). https://doi.org/10.3390/info11020057

Díaz, L. (2017). Soy marca. Profit Editorial. 
Echegaray, L. (2015). Los nuevos roles del usuario: Audiencia en el entorno comunicacional de las redes sociales. En N. Quintas y A. González (Eds.), La participación de la audiencia en la televisión: De la audiencia activa a la social. AIMC.

Fernández-Gómez, J., Hernández-Santaolalla, V. \& Sanz-Marcos, P. (2018). Influencers, marca personal e ideología política en Twitter. Cuadernos.Info, (42), 19-37.

https://doi.org/10.7764/cdi.42.1348

Global Digital Report (2020). Digital in 2020. https://bit.ly/3ga0pMu

Hernández, E. \& Hernández, L. (2018). Manual del comercio electrónico. Marge Books.

Hung, B. W., Jayasumana, A. P. \& Bandara, V. W. (2019). Finding emergent patterns of behaviors in dynamic heterogeneous social networks. Transactions on Computational Social Systems, 6(5), 1007-1019. https://doi.org/10.1109/TCSS.2019.2938787

IAB (2019). Estudio anual de redes sociales 2019. https://bit.ly/2YrS3tk

Ibáñez-Cubillas, P., Díaz-Martín, C. \& Pérez-Torregorsa, A. (2017). Social networks and childhood. New agents of socialization. Education, Health and ICT for a Transcultural World, (237), 64-69. https://doi.org/10.1016/j.sbspro.2017.02.026

Jabłońska, M. R. \& Zajdel, R. (2020). Artificial neural networks for predicting social comparison effects among female Instagram users. PloS One, 15(2), e0229354. https://doi.org/10.1371/journal.pone.0229354

Jin, S.V. \& Ryu, E. (2020). "I'll buy what she's \#wearing": The roles of envy toward and parasocial interaction with influencers in Instagram celebrity-based brand endorsement and social commerce. Journal of Retailing and Consumer Services, (55), 1-15. https://doi.org/10.1016/j.jretconser.2020.102121

Kim, S., Han, J., Yoo, S. \& Gerla, M. (2017). How are social influencers connected in Instagram? International Conference on Social Informatics, 10540, 257-264. https://doi.org/10.1007/978$\underline{3-319-67256-4 \_20}$

Ladogina, A., Samoylenko, I. \& Golovina, V. (2020). Communication effectiveness in social networks of leading universities. Diálogo, (43), 35-50.

https://doi.org/10.18316/dialogo.v0i43.6497

Lee, E., Lee, J. A., Moon, J. H. \& Sung, Y. (2015). Pictures speak louder than words: Motivations for using Instagram. Cyberpsychology. Behavior and Social Networking, 18(9), 552-556. https://doi.org/10.1089/cyber.2015.0157

Marquina-Arenas, J. (2012). Plan social media y community manager. Editorial UOC.

Márquez, I. \& Lanzeni, D. (2018). Media platforms: Instagram. En C. Scolari (Ed.), Teens, media and collaborative cultures. Exploiting teens' transmedia skills in the classroom. CeGe. 
RLCS, Revista Latina de Comunicación Social, 77, 231-252

[Investigación] DOI: 10.4185/RLCS-2020-1456 | ISSN 1138-5820 | Año 2020

Martínez-Sanz, C. \& González-Fernández, R. (2018). Comunicación de marca en Instagram. ¿Una cuestión de género? El rol del influencer de moda. Masculinities and Social Change, 7(3), 230254 https://doi.org/10.17583/MCS.2018.3693

McFarlane, A. \& Samsioe, E. (2020). \#50+ fashion Instagram influencers: Cognitive age and aesthetic digital labours. Journal of Fashion Marketing and Management, 1-16. https://doi.org/10.1108/JFMM-08-2019-0177

Nedra, B., Hadhri, W. \& Mezrani, M. (2019). Determinants of customers' intentions to use hedonic networks: The case of Instagram. Journal of Retailing and Consumer Service, (46), 21-32. https://doi.org/10.1016/j.jretconser.2018.09.001

Niklander, S., Soto, R., Crawford, B., De-la-Barra, C. L. \& Olguin, E. (2016). Towards the easy analysis of celebrity representations through Instagram: A case study. En HCI International $2016-$ Posters' extended abstracts (pp. 67-70). Stephanidis. https://doi.org/10.1007/978-3-319-40542-1_11

Openshaw, J. (2014). The socially savvy advisor. Vladgrin.

Padilla, C. \& Oliver, A. B. (2018). Instagramers e influencers. El escaparate de la moda que eligen los jóvenes menores españoles. Revista Internacional de Investigación en Comunicación aDResearch, 18(18), 42-59. https://doi.org/10.7263/adresic-018-03

Parmelee, J. H. \& Roman, N. (2020). Insta-echoes: Selective exposure and selective avoidance on Instagram. Telematics and Informatics, 52, 1-10. https://doi.org/10.1016/j.tele.2020.101432

Pérez, C. \& Luque, S. (2018). El marketing de influencia en moda. Estudio del nuevo modelo de consumo en Instagram de los millennials universitarios. adComunica, (15), 255-281. https://doi.org/10.6035/2174-0992.2018.15.13

Ponnusamy, S., Iranmanesh, M., Foroughi, B. \& Sean Hyun, S. (2020). Drivers and outcomes of Instagram Addiction: Psychological well-being as moderator. Computers in Human Behavior, (107), 1-11. https://doi.org/10.1016/j.chb.2020.106294

Rietveld, R., Van-Dolen, W., Mazloom, M. \& Worring, M. (2020). What you feel, is what you like influence of message appeals on customer engagement on Instagram. Journal of Interactive Marketing, (49), 20-53. https://doi.org/10.1016/j.intmar.2019.06.003

Sammis, K., Lincoln, C. \& Pomponi, S. (2015). Influencer marketing for dummies. John Wiley \& Sons.

Sanjuán, A., Quintas, N. \& Martínez, S. (2014). Tabvertising. Formatos y estrategias publicitarias en tabletas. Editorial UOC.

Schöps, J. D., Kogler, S. \& Hemetsberger, A. (2019). (De-)stabilizing the digitized fashion market on Instargam-dynamics of visual performative assemblages. Consumption Markets \& Culture, 23(2), 195-213. https://doi.org/10.1080/10253866.2019.1657099

Segarra-Saavedra, J. \& Hidalgo-Marí, T. (2018). Influencers, moda femenina e Instagram: El poder de la influencia en la era 2.0. Revista Mediterránea de Comunicación, 9(1), 313-325. https://www.doi.org/10.14198/MEDCOM2018.9.1.17 
RLCS, Revista Latina de Comunicación Social, 77, 231-252

[Investigación] DOI: 10.4185/RLCS-2020-1456 | ISSN 1138-5820 | Año 2020

Seyfi, M. \& Soydas, A. U. (2017). Instagram stories from the perspective of narrative transportation theory. The Turkish Online Journal of Design, Art and Communication, 7(1), 47-60. https://doi.org/10.7456/10701100/005

Sheldon, P. \& Bryant, K. (2016). Instagram: Motives for its use and relationship to narcissism and contextual age. Computer in Human Behavior, (58), 89-97.

https://doi.org/10.1016/j.chb.2015.12.059

Skjulstad, S. (2018). Vetements, memes, and connectivity: Fashion media in the era of Instagram. Fashion Theory, 24(2), 181-209. https://doi.org/10.1080/1362704X.2018.1491191

Sprout Social (2020). Important Instagram stats you need to know for 2020. https://bit.ly/2ZvnzXZ

Thomas, V. L., Chavez, M., Browne, E. N. \& Minnis, A. M. (2020). Instagram as a tool for study engagement and community building among adolescents: A social media pilot study. Digital Health, 6. https://doi.org/10.1177/2055207620904548

Tiggermann, M. y Zinoviev, K. (2020). The effect of \#enhancement-free Instagram images and hashtags on women's body image. Body Image, (31), 131-138.

https://doi.org/10.1016/j.bodyim.2019.09.004

Tur-Viñes, V., Núñez-Gómez, P. y González-Río, M. J. (2018). Menores influyentes en YouTube. Un espacio para la responsabilidad. Revista Latina de Comunicación Social, (73), 1211-1230. https://doi.org/10.4185/RLCS-2018-1303

Vizcaíno-Verdú, A., Contreras-Pulido, P. \& Guzmán-Franco, M. D. (2019). Lectura y aprendizaje informal en YouTube: El booktuber. Comunicar, (59), 95-104. https://doi.org/10.1916/C59-2019-09

Weismueller, J. Harrigan, P., Wang, S. \& Soutar, G. (2020). Influencer endorsements: How advertising disclosure and source credibility affect consumer purchase intention on social media. Australasian Marketing Journal (AMJ), 1-11. https://doi.org/10.1016/j.ausmj.2020.03.002

Woo, S. H. \& Kim, S. I. (2019). A study on user experience of Instagram IGTV-Focus on fashion beauty contents service. Journal of Digital Convergence, 17(3), 405-411. https://doi.org/10.14400/JDC.2019.17.3.405

Wu, S., Zeng, J. \& Zewen, Z. (2017). A scheme of privacy protection based on genetic algorithm for behavior pattern of social media users. In Proceedings of 2017 11th IEEE international conference on anti-counterfeiting, security, and identification (pp. 40-44). IEEE. https://bit.ly/3daselL

Zolkepli, I., Hasno, H. \& Mukhiar, S. N. S. (2015). Online social network citizen engagement on Instagram crowdsourcing: A conceptual framework. The Electronic Journal of Knowledge Management, 13(4), 283-292. https://bit.ly/35kd76z 


\section{AUTORES:}

\section{Erika-Lucia Gonzalez-Carrion}

Doctoranda del Programa de Doctorado Interuniversitario en Comunicación de las universidades de Cádiz, Huelva, Málaga y Sevilla en la línea de Educomunicación y Alfabetización Mediática (Media Literacy). Máster Interuniversitario en Comunicación y Educación Audiovisual por la Universidad Internacional de Andalucía y la Universidad de Huelva. Licenciada en Comunicación Social por la Universidad Técnica Particular de Loja (Ecuador) y Licenciada en Ciencias de la Educación con mención en Idioma Inglés por la Universidad Nacional de Loja (Ecuador). Traductora para el blog de la Escuela de Autores de la Revista Científica "Comunicar". Docente de la Universidad Nacional de Loja. Ha sido becaria por la Asociación Universitaria Iberoamericana de Postgrados (AUIP) y de la Universidad Internacional de Andalucía.

erika.gonzalez@unl.edu.ec

ORCID: https://orcid.org/0000-0003-3808-5460

Google Académico: https://tinyurl.com/yysrd76j

\section{Ignacio Aguaded}

Catedrático de Universidad del Departamento de Educación de la Universidad de Huelva (España). Presidente del Grupo Comunicar, colectivo veterano en España en Educomunicación, y Editor Jefe de la reconocida revista científica «Comunicar» (indexada en JCR-Q1, top 4\% mundial 2019, Scopus-Q1, top 1\% mundial...). Es además Director del Grupo de Investigación «Ágora», responsable de múltiples investigaciones nacionales e internacionales y de la dirección de numerosas tesis doctorales. Es Director del Máster Internacional Interuniversitario de Comunicación y Educación Audiovisual (UNIA/UHU) y Director del Programa Interuniversitario de Comunicación (US, UMA, UCA y UHU). Premio de Mejor Investigador de la Universidad de Huelva 2015 en Ciencias Sociales. Ha sido Vicerrector de Tecnologías, Innovación y Calidad de la Universidad de Huelva durante siete años (2005-12). Dirección postal: Universidad de Huelva. Facultad de Ciencias de la Educación. 21071 Huelva (España). web institucional: www.uhu.es/ago aguaded@,uhu.es

Î́ndice H: 60.

ORCID: https://orcid.org/0000-0002-0229-1118

Google Académico: https://tinyurl.com/yx9q14tc 